\title{
Low-voltage Power Line Noise Estimation Using Compressed Sensing
}

\author{
Qilin $\mathrm{Li}^{1, \mathrm{a}}$, Li Xiao ${ }^{2, \mathrm{~b}}$, Changsheng Miao ${ }^{1}$, Jinrui Xiang ${ }^{1}$, \\ Zepeng $\mathrm{Hu}^{2}$, Yang Yue ${ }^{2}$, Wan Qun ${ }^{2} *$ \\ ${ }^{1}$ State Grid Sichuan Electrical Energy Company Metering Center, Chengdu, 610072, China \\ ${ }^{2}$ Department of Electronic Engineering, University of Electronic Science and Technology of China, \\ Chengdu, 611731, China \\ aemail: li_qi_lin@163.com, bemail:709507468@qq.com
}

Keywords: Compressive Sensing; Power Carrier Communication; OMP; Harmonic Noise; Impulsive Noise;

\begin{abstract}
Large-amplitude random impulsive noise and harmonic noise have a very serious impact on the quality of low-voltage power line communication. Taking the time-domain sparsity of random impulsive noise and the frequency-domain sparsity of harmonic noise into consideration, we apply Compressive Sensing (CS) theory to reconstruct the noise, and to restrict the power line noise. The simulations show that the orthogonal matching pursuit (OMP) algorithm can effectively reconstruct the random impulse noise and harmonic noise. Applying this method to the research of carrier communication module interchangeability,we can reduce the bit error rate caused by carrier module interchange, and improve the robustness of carrier communication system.
\end{abstract}

\section{Introduction}

Power grid is the most widely distributed network, which covers most areas in the world. There is a unique advantage of using low-voltage power line as a communication channel. However, the noise pollution of power line is quite serious. Especially the variety and vast amount of electrical connection in low-voltage power line seriously affect the power line carrier communication. In practical situation, the carrier communication system needs to satisfy interchangeability, which requires a higher quality of communication. Thus detecting and eliminating noise in the process of transmission grid carrier have important significance to improve the quality of power line carrier communication and carrier communication module compatibility research.

Indeed, both the impulse noise and harmonic noise have a significant impact on power line carrier communication quality. In this paper, we mainly use the theory of compressive sensing [1,2] to reduce the effect of the two kinds of noise on low-voltage power line carrier communication. According to the practical power line communication environment, we simulate the random impulse noise of power line harmonic noise. With the orthogonal matching pursuit (OMP) algorithm [3,4], the reconstruction of the noise signal is achieved under the condition of time-domain sparsity or frequency-domain sparsity $[5,6]$. Due to the inhibition of impulse noise and harmonic noise, low-voltage power line carrier communication performance has been improved.

\section{Low-Voltage Power Line Noise Model}

The noise of grid basically has two kinds. One is artificial noise, such as power line noise generated by lightning. The other is man-made noise,generated from various electrical equipment, transmission equipment, etc. The factor affecting power line communication is mainly man-made noise $[7,8]$. According to the impact on the communication, it can be divided into three categories: background noise [9], random impulsive noise [10], harmonic noise.

Random impulsive noise is a non-Gaussian noise. Statistical models are usually adopted in two ways: empirical model and physical statistical model. Empirical model does not consider the physical characteristics of the noise. It has low computational complexity, and fits for channel noise simulation. Random Impulsive noise is generally generated by electrical access, and the basic pulse 
width does not exceed 3ms. So random pulse signals in the time domain is clearly sparse.

The other kind we want to estimate is harmonic noise. Low-order harmonics component in the power line harmonic noise have a large amplitude, resulting in a major influence on the communication quality, including 3 times, 5 times and 7 times harmonics. Under certain circumstances frequency, harmonic noise is distributed in a specific number of frequency points. It is clearly satisfied sparsity in the frequency-domain.

The sparsity of random pulse signal in the time-domain and harmonic noise in the frequency-domain provides a good foundation for the application of compressive sensing theory.

\section{Low-Voltage Power Line Noise Reconstruction Model}

In compressive sensing, signal $\mathbf{x} \in R^{N}$ can be recovered from the observation $\mathbf{y} \in R^{M}$ with less dimension $(\mathrm{M}<\mathrm{N})$ without loss of the original information. The transform from $\mathbf{x}$ to $\mathbf{s}$ is called sparse representation of signals:

$$
\mathbf{x}=\boldsymbol{\Phi} \mathbf{s} \text {. }
$$

In the type, $\mathbf{s}$ is a sparse signal.

The measurement matrix $\Psi \in R^{M \times N}(M<<N)$ is known, $\mathbf{y}$ is a projection of signal $\mathbf{x}$ in the measurement matrix:

$$
\mathbf{y}=\Psi \mathbf{x}
$$

Combining Eq.1 and Eq.2, y can be further expressed as:

$$
\mathbf{y}=\Psi \mathbf{x}=\Psi \Phi \mathbf{s}=\Theta \mathbf{s}
$$

In the type (3), $\boldsymbol{\Theta}$ is called the observation matrix.

Since $\mathbf{M}<\mathrm{N}$, recovery of the signal $\mathbf{x}$ from the measurements $\mathbf{y}$ is ill-posed. However, the CS theory tells us that when the matrix $\Theta$ has the Restricted Isometry Property (RIP), then we can recover the $\mathrm{K}$ largest element from a similarly sized set of $M=O(K \log (N / K))$ measurements y with high possibility [11,12]. The reconstruction algorithm is broadly divided into three categories: greedy iterative algorithms, convex relaxation algorithms and combinatorial algorithms. Greedy iterative algorithms, such as Orthogonal matching pursuit (OMP), with low computational complexity are easy to realize. In the condition of stochastic impulsive noise with time-domain sparse and harmonic noise with frequency-domain sparse, we only need to find the appropriate observation matrix to reconstruct the noise.

According to the characteristics of power line channel, establish the signal model:

$$
s(n)=x(n)+e(n), \quad n=0,1, \cdots, N .
$$

In the type, $x(n)$ is the original signal; $e(n)$ is the noise signal; $S(n)$ represents the observed signal. Considering the characteristics of power system noise, $e(n)$ is further stated as:

$$
e(n)=i(n)+f(n) .
$$

In the type (5), $i(n)$ and $f(n)$ respectively represent the stochastic impulsive noise and harmonic noise. Bernoulli-Gauss (BG) model is usually used for random impulsive noise simulation: $i(n)=b(n) g(n)$, where $b(n)$ is Bernoulli random sequence which reflects the fact that positions impulsive noise occurrents obey the Bernoulli stochastic process. $g(n)$ is Gauss 
series ,which describes the amplitude satisfies Gauss distribution, and $f(n)$ mainly includes the odd harmonics with large amplitude which seriously affects the communication. According to the sparsity of impulsive noise in time domain and harmonic noise in frequency-domain, we estimate $i(n)$ and $f(n)$ respectively.

According to the time-domain sparsity of random impulsive noise, we design the $M \times N$ observation matrix $\boldsymbol{\Theta}_{i(n)}=\left\{\theta_{1}, \theta_{2}, \cdots, \theta_{N}\right\}$.The number of observations is $M$. We assum that random impulsive noise appears in $K$ sampling points among $M$ observations, and then $\theta_{j}(j=1,2, \cdots N)$ is a sequence of $M$ elements, in which we except $K$ elements is 1 , while other elements are zero. There are $N=C_{M}^{K}$ sequences satisfying this condition, which constitute observation matrix $\boldsymbol{\Theta}_{i(n)}$.In this condition, the collection $\left\{\theta_{j}, j=1,2, \cdots N\right\}$ contains all the possibilities of $K$ positions impulsive noise occurred. Through the OMP algorithm, we get position and amplitude estimation of impulsive noise, thus to realize the reconstruction.

For harmonic noise, we should estimate frequency and phase of each component. Here we only focus on the similarity of harmonic signal reconstructed with original harmonic signal waveform, without considering the fuzzy problem between the phase and frequency. Frequency of harmonic noise is distributed in several specific points, and phase satisfies the $(-\pi, \pi)$ random distribution. Assuming the harmonic noise includes $K$ larger frequency components, and the harmonic noise is a $K$ sparse signal in time-domain, then the construction of the observation matrix is:

$$
\boldsymbol{\Theta}_{f(n)}=\left[\begin{array}{cccc}
\exp \left\{j 2 \pi f_{1} t_{1}\right\} & \exp \left\{j 2 \pi f_{2} t_{1}\right\} & \cdots & \exp \left\{j 2 \pi f_{N} t_{1}\right\} \\
\exp \left\{j 2 \pi f_{1} t_{2}\right\} & \exp \left\{j 2 \pi f_{2} t_{2}\right\} & \cdots & \exp \left\{j 2 \pi f_{N} t_{2}\right\} \\
\vdots & \vdots & \ddots & \cdots \\
\exp \left\{j 2 \pi f_{1} t_{M}\right\} & \exp \left\{j 2 \pi f_{2} t_{M}\right\} & \cdots & \exp \left\{j 2 \pi f_{N} t_{M}\right\}
\end{array}\right] .
$$

In the type, $t_{m}(m=1,2, \cdots M)$ are sampling points; $\left\{f_{n}, n=1,2, \cdots N\right\}$ represents the search range of frequency. The OMP algorithm can obtain the estimation of each harmonic component of noise. However, due to the existence of frequency and phase ambiguity, the estimations of frequency and phase are not necessarily accurate. But this does not affect the reconstruction of harmonic noise, and the reconstruction signal waveform is highly approximate with the original harmonic noise.

\section{Experimental Results}

In order to validate the reconstruction performance of OMP algorithm to random impulsive noise and harmonic noise, we conducted a simulation experiment. In the experiment, the random impulsive noise $i(n)=b(n) g(n)$ is produced by Bernoulli-Gauss model, where $b(n)$ satisfies the Bernoulli random distribution with $\lambda=0.03, g(n)$ is Gauss sequence with mean 30 and variance 10.In addition, we select the sampling points $\mathrm{N}=201$ and the power line frequency $f_{0}=50 \mathrm{~Hz}$.Harmonic noise is considered with the larger amplitude of harmonic components, including 3 times, 5 times,7 times harmonics:

$$
f(n)=5 \times \sin \left(2 \pi \times 3 f_{0} t(n)+\varphi_{3}\right)+5 \times \sin \left(2 \pi \times 5 f_{0} t(n)+\varphi_{5}\right)+5 \times \sin \left(2 \pi \times 7 f_{0} t(n)+\varphi_{7}\right) .
$$

In the type, $t(n)$ represents the $\mathrm{N}$ points time sampling sequence, $\varphi_{i}(i=3,5,7)$ are random phases. Fig.1 shows the power line noise simulation created by the superposition of random impulsive noise and harmonic noise. 


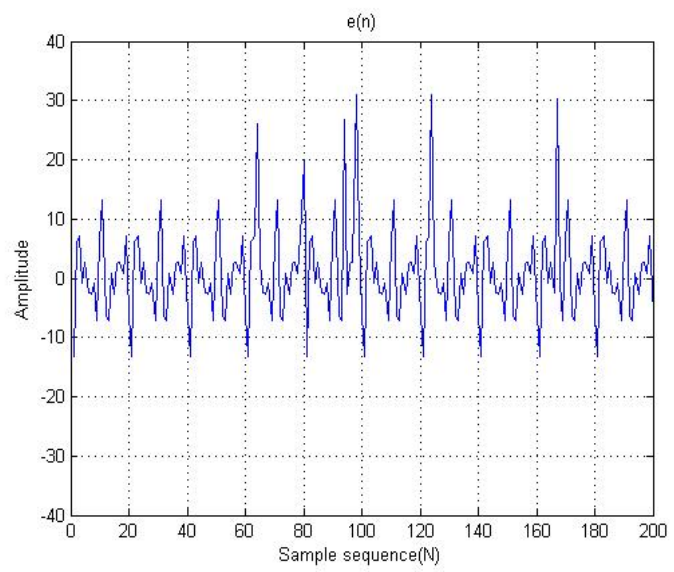

Fig.1 Power line noise
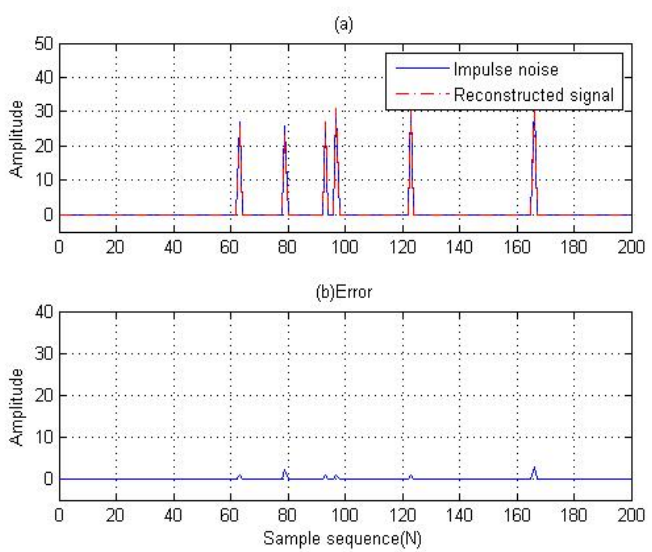

Fig.2 (a) Reconstructed signal of impulsive noise; (b) Error between $\hat{i}(n)$ and $i(n)$

To estimate impulsive noise and harmonic noise, we constructed the observation matrix $\boldsymbol{\Theta}_{i(n)}$ and reconstruct the impulsive noise through the OMP algorithm. In Fig.2, (a) shows the reconstruction of impulsive noise $\hat{i}(n)$. As we can see from (b), the error between $\hat{i}(n)$ and $i(n)$ is very small.

Using the observation matrix $\boldsymbol{\Theta}_{f(n)}$ given by Eq.6, one gets K estimations of the frequency and phase of harmonic components by OMP algorithm to realize the reconstruction of harmonic signal. Fig. 3 shows the reconstruction of the reconstructed harmonic noise $\hat{f}(n)$ and the original harmonic noise $f(n)$.

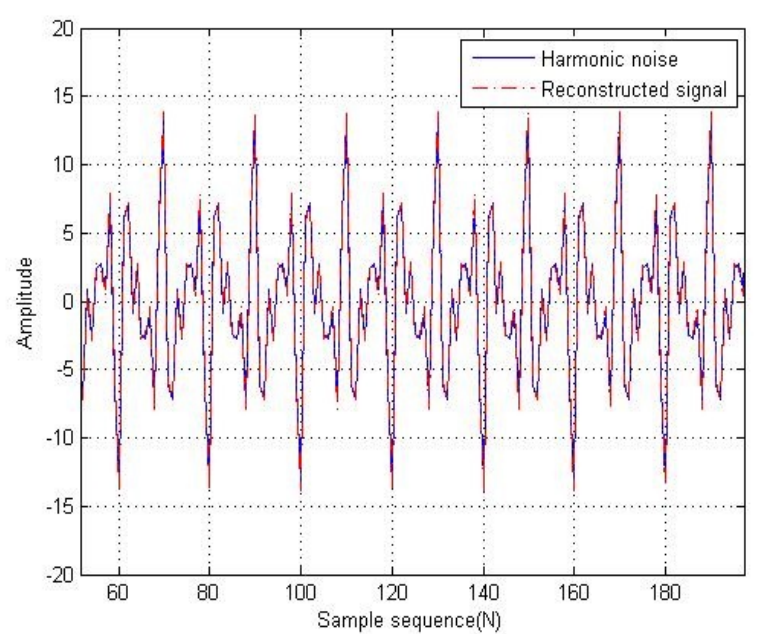

Fig.3 Reconstructed signal of harmonic noise
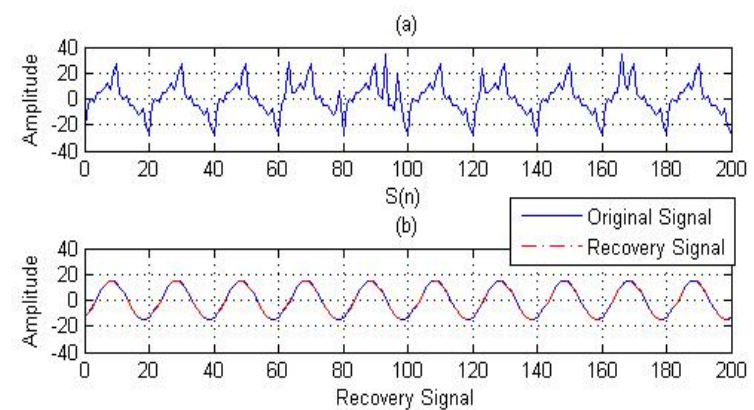

(c)

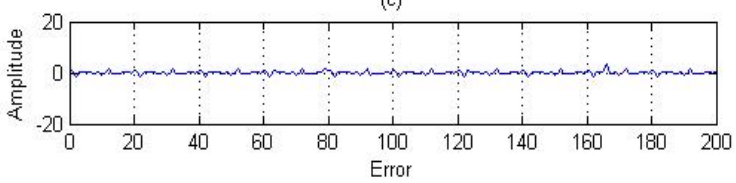

Fig.4 (a) Signal with noise $s(n)$; (b) Recovery signal $\hat{x}(n)$; (c) Error between $\hat{x}(n)$ and $x(n)$

The reconstructed noise $\hat{e}(n)$ can be created by the superposition of reconstructed random impulsive noise $\hat{i}(n)$ and $\hat{f}(n)$ harmonic noise. In order to facilitate the research, one takes sine signal as the input signal with the amplitude 15 and frequency $50 \mathrm{~Hz}$. We can get the signal with noise $s(n)$ by Eq.4. Through the OMP algorithm, we realize the reconstruction of the impulsive noise and harmonic noise separately. Then we can get the noise estimation $\hat{e}(n)$. From Fig.4, we can get the conclusion that the recovery signal $\hat{x}(n)=s(n)-\hat{e}(n)$ is very similar to the original signal, and the error is very small. 


\section{Conclusion}

Random impulsive noise and harmonic noise in the power line are the main causes that affects power line carrier communication. In this article, we analyse the noise in low-voltage power line communication environment, research the feasibility of using CS theory to reconstruct noise with the sparsity of noise in the time domain or frequency domain, and design the corresponding observation matrix. Reconstruction noise is realized by OMP algorithm, which verify the effectiveness of the CS theory in the power line signal noise suppression.

\section{References}

[1] Donoho D L. Compressed sensing[J]. Information Theory, IEEE Transactions on, 2006, 52(4): 1289-1306.

[2] Foucart S, Rauhut H. A mathematical introduction to compressive sensing[M]. Berlin: Springer, 2013,pp. 60-70.

[3] Baraniuk R, Davenport M, DeVore R, et al. A simple proof of the restricted isometry property for random matrices[J]. Constructive Approximation, 2008, 28(3): 253-263.

[4] X.C. Cong, R.Q. Zhu, Y.L. Liu, Q. Wan*, Feature extraction of sar target in clutter based on peak region segmentation and regularized orthogonal matching pursuit, Signal and Information Processing (ChinaSIP), 2014 IEEE China Summit \& International Conference on, pp: 189-193.

[5] Candès E J, Romberg J, Tao T. Robust uncertainty principles: Exact signal reconstruction from highly incomplete frequency information[J]. Information Theory, IEEE Transactions on, 2006, 52(2): 489-509.

[6] Elad M. Sparse and redundant representations: from theory to applications in signal and image processing[M]. Springer, 2010,

[7] Zimmermann M, Dostert K. An analysis of the broadband noise scenario in powerline networks[C]//International Symposium on Powerline Communications and its Applications (ISPLC2000). 2000: 5-7.

[8] Hooijen O G. A channel model for the residential power circuit used as a digital communications medium[J]. Electromagnetic Compatibility, IEEE Transactions on, 1998, 40(4): 331-336.

[9] Zimmermann M, Dostert K. An analysis of the broadband noise scenario in powerline networks[C]//International Symposium on Powerline Communications and its Applications (ISPLC2000). 2000: 5-7.

[10]Hooijen O G. A channel model for the residential power circuit used as a digital communications medium[J]. Electromagnetic Compatibility, IEEE Transactions on, 1998, 40(4): 331-336.

[11]Cho N I, Choi C H, Lee S U. Adaptive line enhancement by using an IIR lattice notch filter[J]. Acoustics, Speech and Signal Processing, IEEE Transactions on, 1989, 37(4): 585-589.

[12]Clancy E A, Morin E L, Merletti R. Sampling, noise-reduction and amplitude estimation issues in surface electromyography[J]. Journal of Electromyography and Kinesiology, 2002, 12(1): $1-16$. 